\title{
FSS on energy saving glass for rf communication enhancement in modern building
}

\author{
Najihah Zakaria, S. N. Azemi, P. J. Soh, C.B.M.Rashidi, A Abdullah Al-Hadi \\ Advanced Communication Engineering Center (ACE), School of Computer and Communication Engineering, \\ University Malaysia Perlis, Malaysia
}

\begin{tabular}{l} 
Article Info \\
\hline Article history: \\
Received Sep 30, 2018 \\
Revised Nov 26, 2018 \\
Accepted Jan 25, 2019 \\
\hline
\end{tabular}

Keywords:

Energy saving glass panel Frequency selective surface Incident angle

RF enhancement

\begin{abstract}
The use of energy saving glass has become very popular in the modern day building design. This energy saving property is achieved by applying a very thin tin oxide ( $\mathrm{SnO} 2)$ coating on one side of the glass. This coating can provide good thermal insulation to the buildings by blocking infrared rays while being transparent to visible part of the spectrum. Drawbacks of these energy saving windows is that it also attenuates the transmission of useful microwave signals through them. These signals fall within the frequency band of $0.8 \mathrm{GHz}$ to $2.2 \mathrm{GHz}$. In order to pass these signals through the coated glass, the use of aperture type frequency selective surface (FSS) has being proposed. In the present work, $\mathrm{SnO} 2$ thin film with FSS structure was fabricated. Microwave transmission through $\mathrm{SnO} 2$ coated glass with FSS structure was also analyzed using network analyzer. The result of computer simulation was confirmed and consistent with the network analyzer results that showed the improvement of $\mathrm{SnO} 2$ coated glass with the FSS structure.
\end{abstract}

Copyright (c) 2019 Institute of Advanced Engineering and Science. All rights reserved.

\section{Corresponding Author:}

Saidatul Norlyana Azemi,

Advanced Communication Engineering Center (ACE),

School of Computer and Communication Engineering,

University Malaysia Perlis, Malaysia.

Email: snorlyana@unimap.edu.my

\section{INTRODUCTION}

The tropical country with hot and wet weather all along the year known as the Malaysia country. Most of the year in Malaysia weather average in $37^{\circ} \mathrm{C}$. Nowadays, modern building will use air conditioning as solution to release the heats to outside. Then, the usage of air conditional will make electrical power consumption will increase. In addition, heavy usage of air conditioning is not good for the mother earth due to depleting of ozone layer. Recently, energy saving glass has been developed to overcome this problem. Energy saving glass could help to reduce the temperature inside the buildings by reflecting the infrared light that penetrates through the building [1-4].

The most basic energy saving glass is a glass that applied with a very thin tin oxide (SNO2) film on it. This SNO2 material is a semiconducting oxide that have higher band gap are suitable in the gas sensor due to the higher free electron in the oxygen vacant holes and thus increased the electrical conductivity of the thin film, solar cell, flat panel display and photo catalysis. However, the disadvantages of the energy saving glass is that reflect the important electromagnetic wave such as GSM mobile signal, GPS, and Bluetooth. In order to improve the electromagnetic signal inside the building, FSS had been added into the energy saving glass. This FSS structure will helps to enhance the electromagnetic wave inside the building. Different FSS structure will give different transmission at various frequency. The optimized FSS structure will give the better transmission in the particular frequency. [5-7].

The use of a very thin metallic coating in modem window design is an extremely effective way to save energy. Acting as a filter, the shielding blocks the electromagnetic radiation in the infrared region and is 
completely transparent to the visible part of the spectrum, thus rejecting the heat outside of a building during summer and keeping the heat inside during winter. These special windows are called low-emissivity (or energy saving) windows since the metallic oxide layer utilized reflects a significant portion of long and short infrared wave energy. From a communication point of view, there is a subsequent problem with the use of such technology, the electromagnetic radiation in the microwave region is blocked and the blocking gets more intense as frequency increases. This means that wireless communication is severely restricted into and out from buildings. Hence, for GSM and Wireless Local Area Networks (LANs) use inside buildings, transparency is needed. A solution to this problem is to create an FSS in the metallic coating of the lowemissivity glass [8].

FSS is a structure that allow the certain frequencies to passed through it while block other frequencies. The used of FSS in this project was to improve the microwave frequencies. In the past few years, many researches had tries to apply different structure on the energy saving glass. Band-pass FSS that act as filter with single, double and triple glass used to improve the transmission of $\mathrm{RF} / \mathrm{microwave}$ frequencies [9-11].The energy saving glass available for four season country is double panels that argon gas was filled in the middle of it and currently none of the research was reported in Malaysia. This energy saving glass is specially designed for four season countries. While Malaysia is a tropical country that only need a single panel energy saving glass. For single glass panel energy glass is relatively cheaper than the double panel energy saving glass that filled with Argon gas. Figure 1 shows and illustration on how a FSS windows work. The presence coating of saves energy glass panel will block the infrared radiation into the buildings and ultimately the load on heating or cooling system is reduced. Unfortunately, this coating also affects the transmission of useful RF and microwave signals in 1-2 GHz band. This attenuation results in poor communication between inside and outside the building. In consequence, a new solution of technologies for modern building is proposed the FSS to overcome this problem. The presence of electromagnetic wave of frequency selective surface will vary the frequency change of FSS due to block signal from outside and inside the building [12].

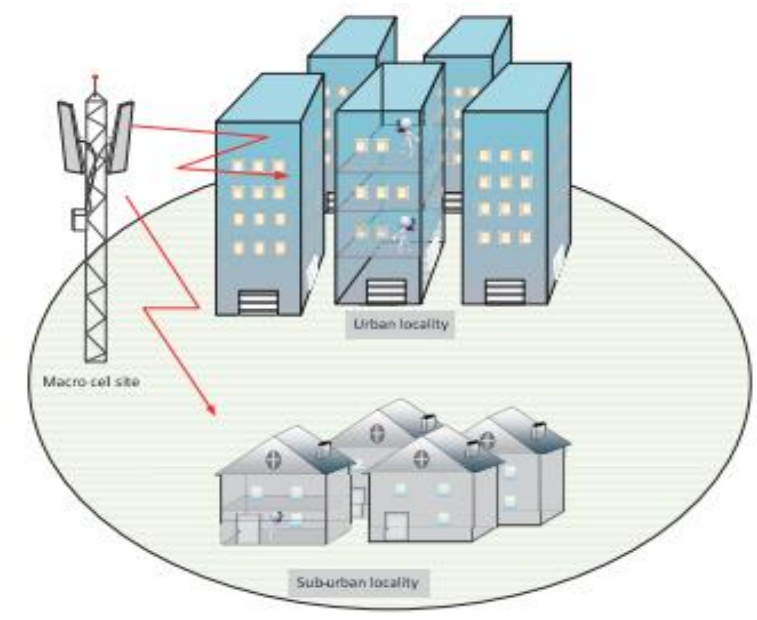

Figure 1. Illustration of how a frequency selective window works

\section{DEVELOPMENT OF DOUBLE SQUARE LOOP FREQUENCY SELECTICE SURFACE}

The elements shape of FSS that were proposed which is a double square loop. This design was proposed to improve the directivity of the directive antenna at operating frequency which $2.45 \mathrm{GHz}$ in this case and belong to band stop frequency respond with S11 higher than $10 \mathrm{~dB}$ S21 less than 40dB. The projected square loop FSS is a periodic array of square loop layer and it have to improve the directivity of the electromagnetic wave as a result of the tapered structure. The TE and TM angular stable is obtained by tapering the width of a cylinder with a square cross-section from the upper opening to the lower opening. Impressive frequency stability and transmission characteristic have to achieve up to a certain range of degrees for both TE and TM incidence angles. The effect of important design parameters on the FSS characteristic has been examined using CST simulation software and will clarified in detail in the next section.

The design the directive antenna using FSS has remarkable expected in solving problem faced by directive antenna using helical and EBG resonator [13-15]. After the background study regarding the FSS 
element shape, the square loop of FSS provides a basic element for this project. The square loop design was chosen as a basic element since it has been verified less sensitivity to incident angle than the other shape elements. Since for useful application the FSS would enlightened by waves of different angular stability, therefore a respectable FSS should have good performances with respect to different situations.

The polarization of the incident signal is also affect the FSS response. Therefore, the wave transmission of the FSS designing will exposed to the different polarization and incident signal. Dual-polarization that involved is included transverse electric (TE) and transverse magnetic (TM) polarizations. They are characteristically separately into the orthogonal TE and TM where TE is an electric field upright to plane of incidence while TM is magnetic field vertical to plane of incident. The dimension of centered connector FSS allows an additional design parameter enables enhanced control of TE and TM responses without the need for external dielectric matching layer. These properties give the absorb/transmit FSS a clear advantage over conventional FSS. In order to obtain a good fundamental centered connector design the initial comprehensive parametric studies were carried out experimentally using CST Microwave Studio Suite simulation software. The calculation is done to determine the size of the element shape to use in designing an FSS. It is depending on the frequency and the thickness of the substrate. The calculation of parameter is shows following as [16]:

a) Calculation of Width, W:

$$
\begin{aligned}
& W=\frac{1}{2 f(\sqrt{\varepsilon \sigma \mu o})} \sqrt{\frac{2}{\varepsilon r+1}} \\
& W=\frac{c}{2 f o \sqrt{\frac{(\varepsilon r+1)}{2}}}
\end{aligned}
$$

$W=34.77 \mathrm{~mm}$

b) Calculation of Effective Dielectric Constant

$$
\begin{aligned}
& \xi e f f=\left(\frac{\varepsilon r+1}{2}\right)+\left[\left(\frac{\xi r+1}{2}\right)\left(1+12 \frac{h}{W}\right)^{-0.5}\right] \\
& \xi e f f=4.78
\end{aligned}
$$

c) Calculation of Line Extension

$$
\Delta L=0.42 h\left(\frac{(\xi e f f+0.3)\left(\frac{W}{h}+0.264\right)}{(\xi e f f-0.258)\left(\frac{W}{h}+0.8\right)}\right)
$$

$\Delta L=0.76 \mathrm{~mm}$

d) Calculation of Length, L

$$
\begin{aligned}
L & =\frac{1}{2 f(\sqrt{\xi e f f} \sqrt{\xi o} \sqrt{\mu o})}-2 \Delta L \\
L & =25.25 \mathrm{~mm}
\end{aligned}
$$

Where:

$$
\begin{array}{ll}
c= & \text { velocity of light in free space } \\
\varepsilon O= & \text { relative permittivity of constant } \\
\varepsilon r= & \text { relative permittivity of substrate } \\
\mu o= & \text { relative permeability of constant } \\
h= & \text { thickness of substrate } \\
f o= & \text { frequency at free space } \\
\tan \delta= & \text { loss tangent }
\end{array}
$$

e) Calculation of Size FSS 


$$
\begin{aligned}
& \lambda=\frac{c}{f}=\frac{3 \times 10^{8}}{2.45 \times 10^{9}}=0.12 \\
& \text { Size FSS }=\frac{\text { Length }}{\lambda}=\frac{26.33 \times 10^{-3}}{0.12}=219.42 \mathrm{~mm}^{2}
\end{aligned}
$$

Where: $\lambda=$ wavelength

$$
c=\left(3 \times 10^{8}\right) \text { Speed of light }
$$

$f=$ frequency, $\mathrm{Hz}$

The square loop FSS design was created by reasoning the height of planar square loop unit cell. This change of the loop in to square element of a define length. The modification to this length changes the frequency response characteristic of FSS, allowing a better angular response to be accomplished, in order to improve the directivity of the directive antenna; the structure is modified by tapering the square loop element of the superstrate. By creating a 2D structure, it proposed that FSS will provide better transmission for a narrow range of bandwidth. The Table 1 is the transmittance from the cut-double square loop elements to improve that the return loss is less $10 \mathrm{~dB}$ at $2.45 \mathrm{GHz}$. As shown Figure 2 the fss double square loop design.

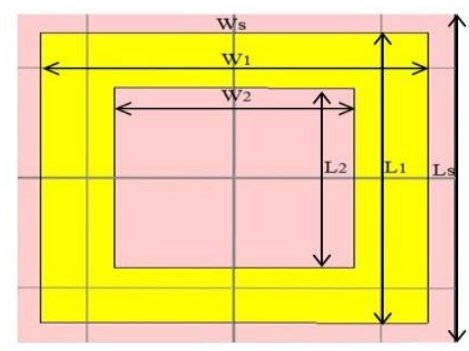

Table 1. The List of Parameters of FSS Design

\begin{tabular}{cc}
\hline Parameter & Symbol \\
\hline Gap & $\mathrm{G}$ \\
Height of Substrate & $\mathrm{H}$ \\
Length of Inner Loop & $\mathrm{L}_{1}$ \\
Length of Inner Loop & $\mathrm{L}_{2}$ \\
\hline
\end{tabular}

Figure 2. The fss double square loop design

\section{RESULT AND DISCUSSION}

With the previous section, the theory of FSS, development and the fabrication process of the FSS on glass substrates were discussed in detail. Here, all the results of the FSS on glass substrate were obtained from the simulation and measurement process that being done throughout this project were being recorded, analyzed and discuss. Configuration of this section is divided into two parts which are simulation result and measurement result. From the simulation, S11 and S21 were discussing as both parameters indicate frequency drop and magnitude respectively.

The outlines of this chapter consist of:

a. S11 and S21 simulation results of Circular Ring and Square Loop design

b. S11 and S21 measurement results of Circular Ring and Square Loop design

c. S11 and S21 comparison results of Circular Ring for type FR-4 and Glass Pyrex

d. S11 and S21 comparison results of Square Ring for type FR-4 and Glass Pyrex.

\subsection{Circular Ring Design For Fr-4}

As shown in Figure 3 is the design of Circular Ring with its parameter and Figure 4 shows the simulation results of S11 and S21. As shown in Figure 4, the Circular Ring FSS designed in this section is a band pass filter with the initial height of copper which is $0.035 \mathrm{~mm}$ and the operating frequency at $2.45 \mathrm{GHz}$. The surface of the structure transmits signals at frequencies lower or higher than $2.45 \mathrm{GHz}$, which is act as band stop respond but reflects the signal at $2.49 \mathrm{GHz}$ with $-10 \mathrm{~dB}$. In this case, the frequency responses at -10 $\mathrm{dB}$ are between $2.34 \mathrm{GHz}$ and $2.65 \mathrm{GHz}$, therefore the bandwidth is $12.34 \%$ approximately. Parameters for circular ring FSS design as shown in Table 2.

Table 2. Parameters for Circular Ring FSS Design

\begin{tabular}{ccc}
\hline Parameters & Description & Dimension $(\mathrm{mm})$ \\
\hline W & Unit cell size & 40 \\
O_ring & Outer square length & 32 \\
I_ring & Inner square length & 28 \\
z2 & Thickness FSS & 0.035 \\
S & Spacing between square shape & 4 \\
\hline
\end{tabular}




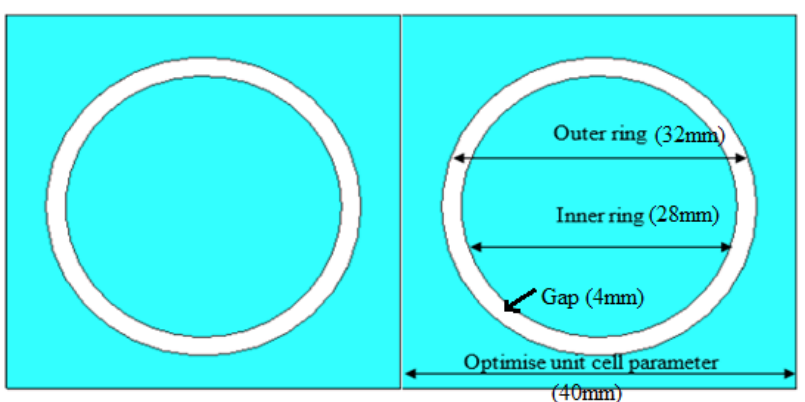

Figure 3. Unit cell parameter were changed for simulation

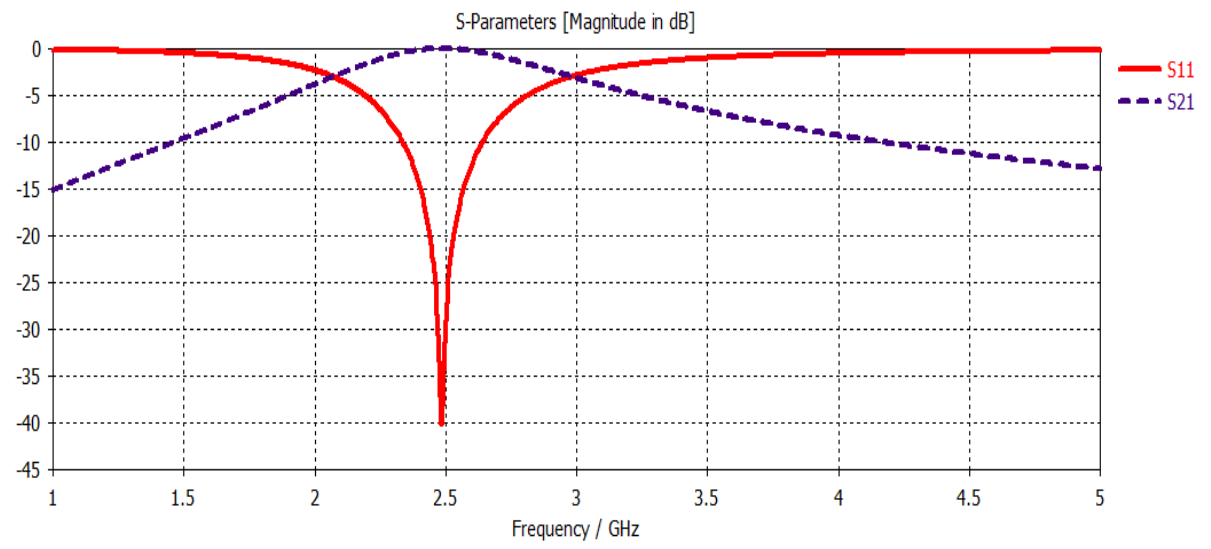

Figure 4. S11 and S21 simulation result

\subsection{Circular Ring Design For Glass Pyrex}

As shown in Figure 5 is the design of Circular Ring with substrate glass pyrex with its parameter and Figure 6 shows the simulation results of S11 and S21. Parameters for circular ring FSS design as shown in Table 3.

Table 3. Parameters for Circular Ring FSS Design

\begin{tabular}{ccc}
\hline Parameters & Description & Dimension $(\mathrm{mm})$ \\
\hline W & Unit cell size & 40 \\
O_ring & Outer square length & 32 \\
I_ring & Inner square length & 28 \\
z2 & Thickness FSS & 0.035 \\
S & Spacing between square shape & 4 \\
\hline
\end{tabular}

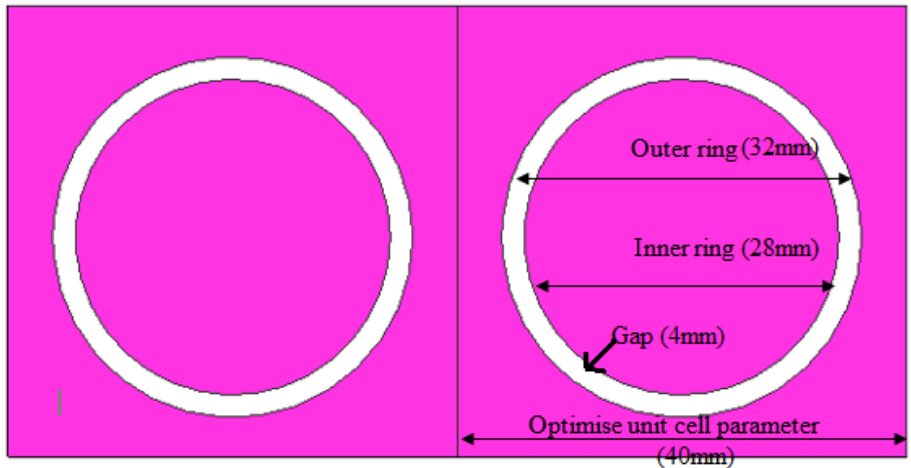

Figure 5. Unit cell parameter were changed for simulation 


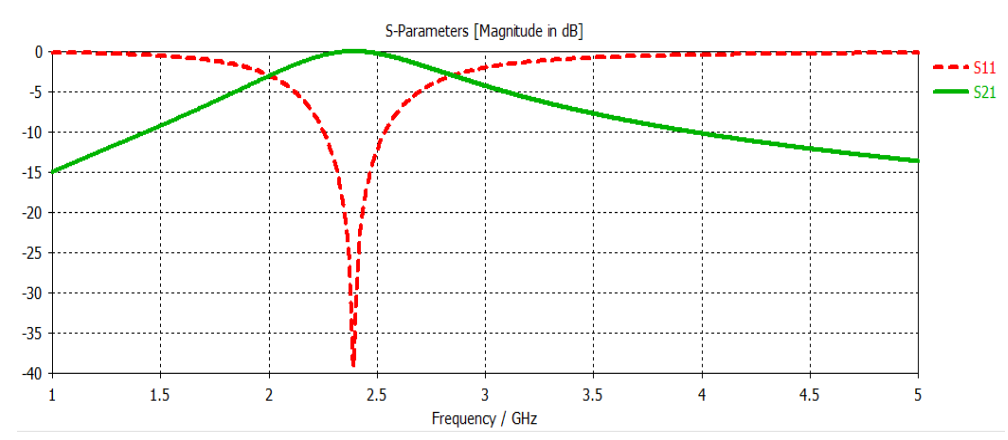

Figure 6. S11 and S21 simulation result

As shown in Figure 6, the Circular Ring FSS substrate Glass Pyrex designed in this section is a band pass filter with the initial height of copper which is $0.035 \mathrm{~mm}$ and the operating frequency at $2.45 \mathrm{GHz}$. The surface of the structure transmits signals at frequencies lower or higher than $2.45 \mathrm{GHz}$, which is act as band stop respond but reflects the signal at $2.39 \mathrm{GHz}$ with $-10 \mathrm{~dB}$. In this case, the frequency responses at -10 $\mathrm{dB}$ are between $2.61 \mathrm{GHz}$ and $2.54 \mathrm{GHz}$, therefore the bandwidth is $11.72 \%$ approximately.

\subsection{Comparison Circle Fr-4 And Glass Pyrex}

The simulation has been run under frequency from $1 \mathrm{GHz}$ to $5 \mathrm{GHz}$. It can be concluded from Figure 7 that the simulation result of return loss S11 and transmission S21 response of this two-different type. For S11 Circle FR-4 it drops at $2.49 \mathrm{GHz}$ at $-39.37 \mathrm{~dB}$ while Circle Glass Pyrex drop at 2.39 at $-40.43 \mathrm{~dB}$. The bandwidth for FR-4 is $12.34 \%$ and Glass Pyrex is $11.72 \%$.

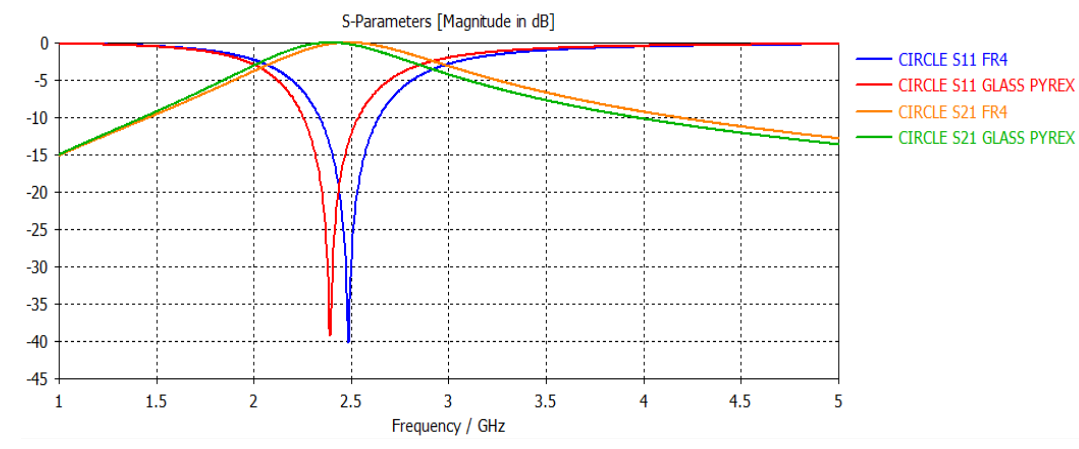

Figure 7. Comparison S11 and S21 simulation result for FR-4 and Glass Pyrex

\subsection{Square Loop Design For Fr-4}

As shown in Figure 8 is the design of Square Ring with substrate FR-4 with its parameter and Figure 9 shows the simulation results of $S 21$ and $S 11$. As shown in Figure 9, the Square Ring FSS designed in this section is a band pass filter with the initial height of copper which is $0.035 \mathrm{~mm}$ and the operating frequency at $2.45 \mathrm{GHz}$. The surface of the structure transmits signals at frequencies lower or higher than 2.45 $\mathrm{GHz}$, which is act as band stop respond but reflects the signal at $2.47 \mathrm{GHz}$ with $-10 \mathrm{~dB}$. In this case, the frequency responses at $-10 \mathrm{~dB}$ are between $2.15 \mathrm{GHz}$ and $2.79 \mathrm{GHz}$, therefore the bandwidth is $25 \%$ approximately. Parameters for circular ring FSS design as shown in Table 4.

Table 4. Parameters for Circular Ring FSS Design

\begin{tabular}{ccc}
\hline Parameters & Description & Dimension $(\mathrm{mm})$ \\
\hline W & Unit cell size & 40 \\
O_ring & Outer square length & 30 \\
I_ring & Inner square length & 26 \\
z2 & Thickness FSS & 0.035 \\
S & Spacing between square shape & 4 \\
\hline
\end{tabular}




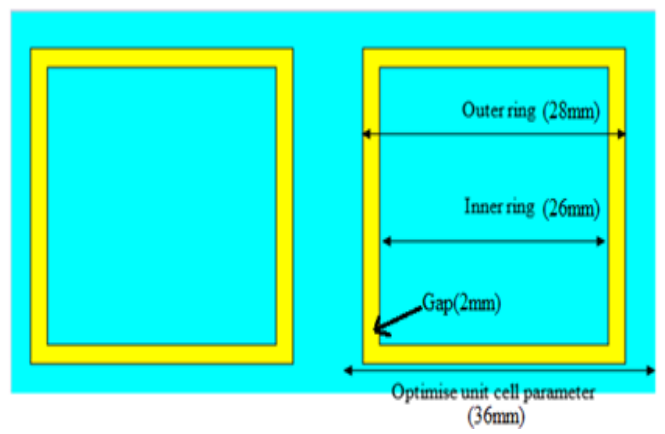

Figure 8. Unit cell parameter were changed for simulation

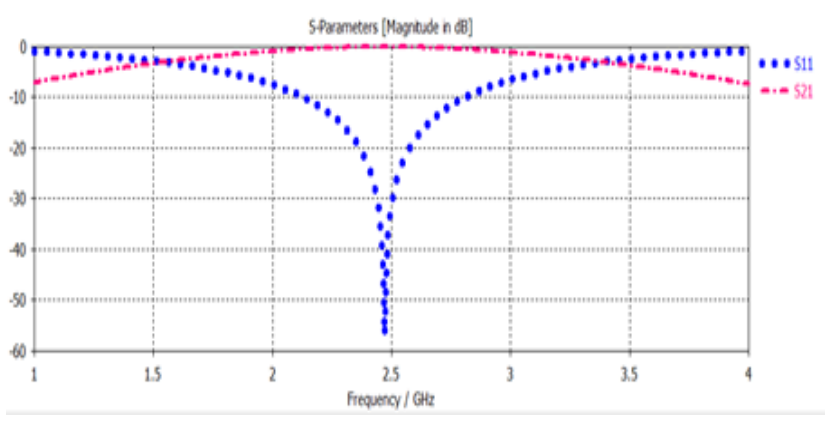

Figure 9. S11 and S21 simulation result

\subsection{Square Loop Design For Glass Pyrex}

As shown in Figure 10 is the design of Square Ring with substrate Glass Pyrex with its parameter and Figure 11 shows the simulation results of $S 21$ and $S 11$. As shown in Figure 11, the Square Ring FSS designed in this section is a band pass filter with the initial height of copper which is $0.035 \mathrm{~mm}$ and the operating frequency at $2.45 \mathrm{GHz}$. The surface of the structure transmits signals at frequencies lower or higher than $2.45 \mathrm{GHz}$, which is act as band stop respond but reflects the signal at $2.40 \mathrm{GHz}$ with $-10 \mathrm{~dB}$. In this case, the frequency responses at $-10 \mathrm{~dB}$ are between $2.06 \mathrm{GHz}$ and $2.73 \mathrm{GHz}$, therefore the bandwidth is $27 \%$ approximately. Parameters for circular ring FSS design as shown in Table 5.

Table 5. Parameters for Circular Ring FSS Design

\begin{tabular}{ccc}
\hline Parameters & Description & Dimension $(\mathrm{mm})$ \\
\hline W & Unit cell size & 40 \\
O_ring & Outer square length & 30 \\
I_ring & Inner square length & 26 \\
z2 & Thickness FSS & 0.035 \\
S & Spacing between square shape & 4 \\
\hline
\end{tabular}

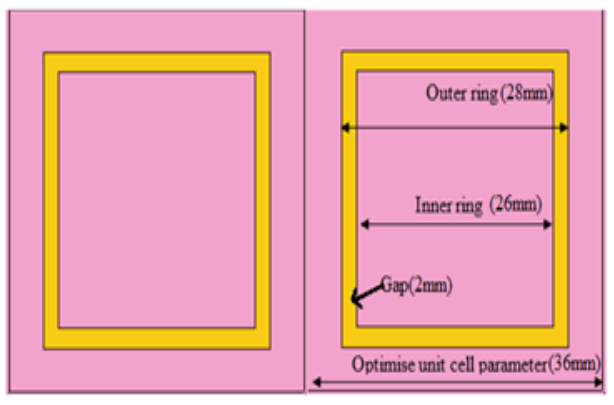

Figure 10. Unit cell parameter were changed for simulation

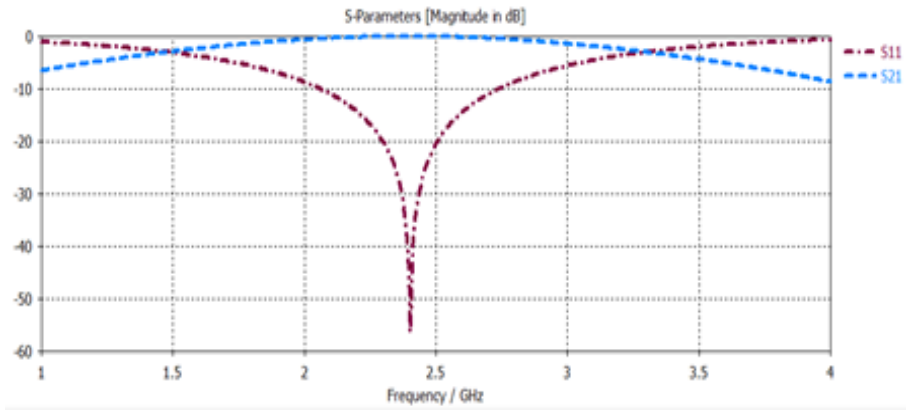

Figure 11. S11 and S21 simulation result

\subsection{Comparison Square Fr-4 And Glass}

The simulation has been run under frequency from $1 \mathrm{GHz}$ to $4 \mathrm{GHz}$. It can be concluded from Figure 4. 10 that the simulation result of return loss S11 and transmission S21 response of this two different type. For S11 Square FR-4 it drop at $2.47 \mathrm{GHz}$ at $-56.31 \mathrm{~dB}$ while Square Glass Pyrex drop at 2.40 at -56.66 $\mathrm{dB}$. The bandwidth for FR-4 is 25\% and Glass Pyrex is 27\%. Comparison S11 and S21 simulation result for FR-4 and glass pyrex as shown in Figure 12. 


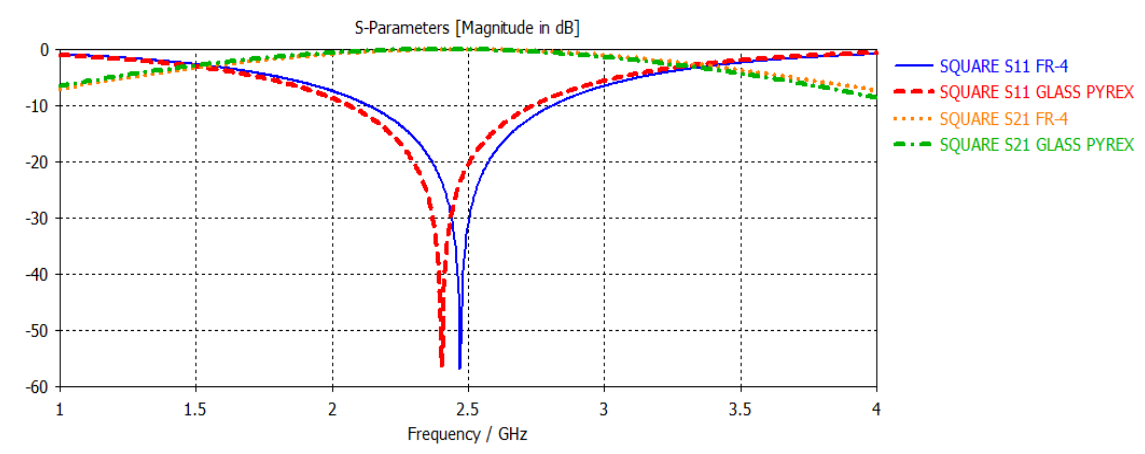

Figure 12. Comparison S11 and S21 simulation result for FR-4 and glass pyrex

\section{CONCLUSION}

Frequency Selective Surfaces (FSS) are planar of periodic structures that behave like a filters to electromagnetic energy. FSS are usually formed by a periodic array of a radiating elements. Generally FSS is a 2D planar structure consisting of one or more metallic patterns, each of it backed by a dielectric substrate. These structures are usually arranged in a periodic fashion and their frequency response is entirely determined by the geometry of the structure in one period called a unit cell. Different elements shape of FSS offer different frequency response and they are divided into four categories which are high-pass, low-pass, band-pass and band-stop filters.

In this project the elements that been chosen are the square and circle elements shape as its gives the best performance in frequency characteristic and has simple shape for the fabrication process. A comprehensive study on the previous methods in designing Frequency selective surfaces revealed that there are some disadvantages and problems with the current FSS. Nevertheless, FSS on the energy saving glass substrate has shown a superior performance then the current FSS. With this kind of background, the design of the new frequency selective surface was started with the goal in producing a new FSS on the glass substrate and to evaluate the frequency selective surfaces design measurements with the simulation and with the fabrication result.

The technical knowledge is included the knowledge of how to use the suitable software to design the FSS and the software are the CST software. Aside from that the mathematical knowledge are also important as it play the most role in the design, as to obtain all the parameters for the circle and square shape such as how much the diameters that can be used to obtain the frequency $2.45 \mathrm{GHz}$. From this projects it's demonstrated the linear relations between the diameters or length of the square with the frequency shifting.

\section{ACKNOWLEDGEMENTS}

The authors gratefully acknowledge use of the services and facilities of the Advanced Communication Engineering Centre (ACE) CoE, School of Computer and Communication Engineering, Universiti Malaysia Perlis (UniMAP). This project also been funded by Fundamental Research Grant Scheme (FRGS) 9003-00545.

\section{REFERENCES}

[1] Xu, Qiao, Xianglei Liu, and Yimin Xuan. "Transparent energy-saving glass with high resistance to solar heat." Journal of Photonics for Energy 9, no. 3, 2018.

[2] Hassan, Nornikman, Zahriladha Zakaria, Badrul Hisham Ahmad, Naim Che Pee, Siti Nadzirah Salleh, Mohamad Zoinol Abidin Abd Aziz, Mohd Fareq Abdul Malek, and Mohd Khairy Ismail. "Number of Iteration Analysis for Complex FSS Shape Using GA for Efficient ESG." Bulletin of Electrical Engineering and Informatics 7, no. 4, 2018.

[3] Kumar, Kiran, Shaik Saboor, Vanish Kumar, Ki-Hyun Kim, and Ashok Babu TP. "Experimental and Theoretical Studies of Various Solar Control Window Glasses for the Reduction of Cooling and Heating loads in Buildings across Different Climatic Regions." Energy and Buildings, 2018.

[4] Chen, Yen-Hsiang, Li-Hung Liao, and Yu-Bin Chen. "Realization of energy-saving glass using photonic crystals." Frontiers in Energy 12, no. 1 (2018): 178-184.

[5] Hussin, F. A., B. H. Ahmad, M. Z. A. A. Aziz, and M. K. Suaidi. "Design and Analysis of Frequency Selective Surface (FSS) Using Complementary Techniques on Glass." Journal of Telecommunication, Electronic and Computer Engineering (JTEC) 10, no. 2-8, 145-150, 2018. 
[6] Lim Huey Sia."Fabrication Of Frequency Selective Structure And Evaluation Of Microwave Transmission On Energy Saving Glass,"Degree of Master Electrical Engineering with Honors, Faculty of Electrical and Electronic Engineering, Universiti Tun Hussein On Malaysia, Johor,Malaysia,(2015).

[7] I. Ullah, D. Habibi, And G. Kiani, "Design Of Rf/Microwave Efficient Buildings Using Frequency Selective Surface,” 2011 Ieee 22nd Int. Symp. Pers. Indoor Mob. Radio Commun., pp. 2070-2074, 2011.

[8] S. I. Sohail, K. P. Esselle, and G. Kiani, "Design of a bandpass FSS on dual layer energy saving glass for improved RF communication in modern buildings," IEEE Antennas Propag. Soc. AP-S Int. Symp., pp. 1-2, 2012.

[9] Azemi, Saidatul Norlyana, Kamran Ghorbani, and Wayne ST Rowe. "3D frequency selective surfaces." Progress In Electromagnetics Research 29, 191-203, 2012.

[10] Azemi, Saidatul Norlyana, and Wayne ST Rowe. "Development and analysis of 3D frequency selective surfaces." In Microwave Conference Proceedings (APMC), 2011 Asia-Pacific, pp. 693-696. IEEE, 2011.

[11] Azemi, Saidatul Norlyana, Kamran Ghorbani, and Wayne ST Rowe. "3D frequency selective surface with incident angle independence." In Microwave Conference (EuMC), 2013 European, pp. 928-931. IEEE, 2013.

[12] Suhaimi, Syaiful Anas, Saidatul Norlyana Azemi, and Ping Jack Soh. "Feasibility Study of Frequency Selective Surfaces for Structural Health Monitoring System." Progress In Electromagnetics Research 80, 199-209, 2018.

[13] Maci, Stefano, Marco Caiazzo, Alessio Cucini, and Massimiliano Casaletti. "A pole-zero matching method for EBG surfaces composed of a dipole FSS printed on a grounded dielectric slab." IEEE Transactions on Antennas and Propagation 53, no. 1, 70-81, 2005.

[14] Lee, Young Ju, Junho Yeo, Raj Mittra, and Wee Sang Park. "Design of a high-directivity Electromagnetic Band Gap (EBG) resonator antenna using a frequency-selective surface (FSS) superstrate." Microwave and Optical Technology Letters 43, no. 6, 462-467, 2004.

[15] Ge, Zhi-Chen, Wen-Xun Zhang, Zhen-Guo Liu, and Ying-Ying Gu. "Broadband and high-gain printed antennas constructed from Fabry-Perot resonator structure using EBG or FSS cover." Microwave and Optical Technology Letters 48, no. 7,1272-1274, 2006.

[16] Munk, B.A., 'Frequency selective surfaces; theory and design'; 2005: Wiley- Interscience. 